\section{㩆 \\ RMA}

Antropología Social

\title{
Michel Foucault, la antropología y el problema del poder
}

\author{
Marc Abélès*
}

*Director de Estudios en la École des Hautes Études en Sciences Sociales (EHESS) e investigador del Centre National de la Recherche Scientifique (CNRS - Laboratorio de Antropología de las Instituciones y las Organizaciones Sociales (LAIOS), IIAC, París.

\section{Traducción de Carolina Villada Castro}

Profesional en filosofía, Universidad de Antioquia, Colombia. Estudiante de la Maestría en Estudios de la traducción, UFSC-PGET, Brasil. E-mail: carolina.villadacastro@gmail.com

N. del T. Marc Abélès, «Michel Foucault, I'anthropologie et la question du pouvoir», L'Homme n 187-188: 105-122. Agradecemos al autor y a la revista la posibilidad y autorización para publicar esta traducción al español. El texto original tiene libre acceso en: https://www.cairn.info/revueI-homme-2008-3-page-105.htm

\begin{abstract}
Résumé
De part et d'autre de l'Atlantique, l'œuvre de Michel Foucault a été reçue de manière très différente par les anthropologues. Si l'anthropologie américaine a accueilli avec enthousiasme les réflexions du philosophe sur les rapports entre pouvoir et savoir, sur sa conceptualisations du biopolitique, les anthropologues français se sont montrés plus réticents et ont manifesté longtemps une certaine méfiance à l'égard de ce penseur. Chez Foucault lui même, on ne trouve que de rares références à l'anthropologie, et son point de vue sur l'anthropologie structurale est extrêmement critique. Cet article est centré sur la question du pouvoir, car c'est là que l'influence foucaldienne sur la nouvelle anthropologie est particulièrement manifeste, et il s'inscrit dans le cadre d'une interrogation plus large sur les rapports entre philosophie et anthropologie.
\end{abstract}

Mots clés: Michel Foucault; anthropologie et philosophie; biopolitique; anthropologie du politique.

\section{Resumen}

A ambos lados del Atlántico, la obra de Michel Foucault ha sido recibida de manera muy diferente por los antropólogos. Si la antropología norteamericana ha acogido con entusiasmo las reflexiones del filósofo sobre las relaciones entre poder y saber, sobre sus conceptualizaciones de lo biopolítico, los antropólogos franceses se han mostrado más reticentes y han manifestado durante mucho tiempo una cierta desconfianza respecto de este pensador. En la obra misma de Foucault, sólo se encuentran escasas referencias a la antropología, y su punto de vista sobre la antropología estructural es extremadamente crítico. Este artículo está centrado en el problema del poder, pues es allí que la influencia foucaultiana sobre la nueva antropología es particularmente manifiesta, y se inscribe en el marco de una interrogación más amplia sobre las relaciones entre filosofía y antropología.

Palabras clave: Michel Foucault; antropología y filosofía; biopolítica; antropología de lo político.

...Y como no tenemos una teoría general para aprehenderlas, soy, si se quiere, un empirista ciego, es decir que estoy en la peor de las situaciones. No tengo una teoría general y no tengo tampoco un instrumento seguro. Ando a tientas, fabrico, como puedo, instrumentos que se destinan a poner de relieve objetos. Los objetos están en alguna medida determinados por los instrumentos buenos o malos que fabrico. Son falsos, si mis instrumentos son falsos...

(Foucault, 2012: 74)

Para muchos antropólogos, los trabajos de Foucault se

${ }^{1} \mathrm{~N}$. del T. Todas las citas referidas se tomaron de las ediciones ya disponibles en español. En los casos de textos sin traducción disponible, proponemos nuestra traducción directa desde el francés. tornaron una referencia imprescindible. Pero es preciso advertir que su influencia se ejerce principalmente al otro lado del Atlántico, donde Foucault ocupa un buen lugar en la enseñanza impartida por los departamentos de antropología de las universidades norteamericanas. Al contrario, en Francia, los antropólogos han manifestado durante mucho tiempo una cierta desconfianza respecto del filósofo. Vale la pena preguntarse por esta diferencia de recepción y recontextualizarla respecto a dos tradiciones intelectuales diferentes.

Si en Francia la antropología se construye en una relación de desconfianza respecto de la filosofía, en los Estados Unidos el desarrollo de una antropología crítica a partir de los años 
1980 ha estado marcado por un interés muy fuerte por la filosofía contemporánea, especialmente por la Escuela de Frankfurt, y los "post-estructuralistas", principalmente Jacques Derrida y Michel Foucault, y más recientemente Giorgio Agamben. Pero sobre todo es interesante analizar la manera en que los antropólogos norteamericanos han recurrido al concepto de poder tal como es trabajado por Foucault. Veremos cómo la reflexión sobre la posición del antropólogo, su "autoridad" como autor, ha venido a encontrarse con las interrogaciones foucaultianas sobre el poder y la noción de disciplina. En otro nivel, nos interesará el impacto de los análisis del filósofo francés sobre la biopolítica, que hoy son una referencia muy presente en la antropología norteamericana y que influencian igualmente los trabajos de los antropólogos franceses. En suma, se indagará sobre las perspectivas que abre la lectura de Foucault a la antropología política tanto a nivel de los desarrollos teóricos como en las prácticas de campo.

Evocar la admisión o el rechazo de la obra de Foucault en el campo de la antropología es, en primer lugar, formular la pregunta más general sobre las relaciones entre este último y la filosofía. A diferencia de una visión simplista que opondría dos problemáticas, una orientada por preguntas fundamentales sobre la naturaleza y los fines de la humanidad, otra llevada por una exigencia de cientificidad y centrada en el conocimiento de las sociedades humanas, hay que reconocer que hace mucho tiempo es una relación compleja y ambigua la que se ha establecido entre antropología y filosofía. Como lo he señalado en otro momento (Abélès, 2005), una de las mayores problemáticas de la antropología naciente, aquella del origen del Estado y, más allá, la gran división entre civilizado y primitivo que ella instaura, se constituyó a partir de una lectura crítica de los filósofos. Uno de los textos fundadores de la antropología política, Ancient Law de Henry Sumner Maine, consagra un capítulo entero a la discusión de las teorías filosóficas del estado de naturaleza y del contrato social, se dedica a una severa refutación de las tesis rousseaunianas, antes de introducir una oposición entre "organización social" donde domina el parentesco y "organización política", fundada sobre el territorio y la propiedad. La división introducida por Maine ha tenido efectos positivos, suscitó una línea de investigación fecunda sobre el parentesco y ha permitido poner en evidencia la consistencia de lo político en las sociedades no estatales. Pero esto refleja también una obsesión de división entre "Nosotros" -los Modernos, aptos para desarrollar un conocimiento objetivo-y "Ellos", estas sociedades-objetos de nuestra ciencia y condenadas a la otredad de lo primitivo y de lo exótico.

Como se observa, hay en este rechazo inaugural del discurso de los filósofos la asunción simultánea de una filosofía, tan implícita como sea, que asigna su lugar al sabio y a los pueblos que serán la materia de sus investigaciones, que le ofrecerán un laboratorio vivo. No es casual que Alfred Radcliffe-Brown, uno de los maestros de la escuela anglosajona, defina a su disciplina como la "ciencia natural de la sociedad humana"; él marca de algún modo un territorio a ocupar y a salvaguardar contra los sortilegios de la especulación. La filosofía y los debates que ella ha podido hacer nacer no pueden más que introducir un desorden inaceptable en un universo donde en adelante los hechos deben reinar.

Esta especificación de la antropología como ciencia natural se inscribe en el prefacio a "una obra de referencia", a decir de sus propios autores, Edward Evans-Pritchard y Meyer Fortes (1940; 2010: 43), sobre los sistemas políticos africanos. De hecho, este libro hizo época: marca un momento esencial en el desarrollo de la antropología política. Es interesante observar que, en su introducción, los autores consagran algunas reflexiones a la filosofía política. Pero es para descalificar enseguida esta aproximación que privilegia el ideal en detrimento de las realidades: "Las teorías de los filósofos políticos no nos han ayudado a comprender las sociedades que hemos estudiado y las consideramos de escaso valor científico, debido a que sus conclusiones raras veces se formulan en términos de comportamiento observado o no son susceptibles de ser comprobadas con este criterio" (ibíd:: 65). Los filósofos han intentado, por cierto, apoyar sus teorías utilizando los datos disponibles sobre las sociedades y las costumbres primitivas, pero en una época en la que esos datos eran aún muy deficientes.

Así los antropólogos deben "evitar toda referencia a lo escrito por filósofos políticos" (ibíd.: 64). El diagnóstico es claro: respecto del desarrollo de la antropología política, la filosofía tiene el aspecto de obstáculo epistemológico. Se puede considerar en última instancia a ciertos filósofos, Montesquieu en particular, como lejanos precursores; pero la perspectiva antropológica implica en su principio una ruptura con el discurso filosófico. Es al menos lo que se desprende de los escritos de Evans-Pritchard y Fortes y de sus discípulos. Este punto de vista ha prevalecido desde entonces, al punto de repercutir sobre nuestra concepción misma de la historia de la antropología. De la misma manera, la refutación de la filosofía parece el corazón mismo del discurso antropológico. Es interesante observar la desconfianza que Claude Lévi-Strauss catedrático que, como se sabe, comenzó su carrera como profesor de filosofía- ha expresado siempre respecto de sus contemporáneos filósofos. Mientras manifiesta una verdadera curiosidad respecto del desarrollo de las ciencias contemporáneas, no dudando trabajar con matemáticos y lingüistas, parece al menos poco interesado por los trabajos de los filósofos.

En las horas gloriosas del estructuralismo, se amalgaman rápidamente pensamientos tan diferentes como los de Roland Barthes, Michel Foucault, Jacques Lacan, Claude Lévi-Strauss. Es poco decir que este último se irritó. Siempre se cuidó de afirmar la especificidad de su enfoque. Por otra parte, no se encontrará en su obra ninguna alusión a los trabajos de Foucault. Habiendo seguido yo mismo su enseñanza (él fue mi director de tesis), pude observar 
la desconfianza de la que daba prueba con respecto a los enunciados filosóficos. Si ha aceptado discutir con Paul Ricoeur, no se dignó nunca a comentar el texto que Derrida consagró al capítulo de Tristes trópicos titulado "Lección de escritura" (Derrida, 1986: 133-180). Respecto a la influencia de Lévi-Strauss en el desarrollo de la antropología francesa, no sorprenderá que los filósofos en general, y Foucault en particular, hayan sido de algún modo considerados como fuera de campo. Esto puede parecer tanto más curioso ya que muchos antropólogos antes habían estudiado filosofía. Pero, precisamente, se trataba de romper con la especulación y de comprometerse en un paciente trabajo de recolección de datos, yendo sobre el terreno. El terreno constituía el medio de acceder a lo concreto, pero también hacía el papel de rito de pasaje por el cual se suponía purificado del etnocentrismo dominante.

En ese contexto, la filosofía aparecía en el mejor de los casos como un lujo inútil; en el peor, como una serie de prejuicios inadecuados. No sorprenderá entonces que no se encuentre ninguna alusión a Foucault o a Derrida en los trabajos más destacados producidos por la generación post-levistraussiana que paradójicamente había vivido la época del estructuralismo triunfante y había podido seguir las enseñanzas de Foucault y Derrida. ¿Hay que hablar de anatema respecto de la filosofía? En cualquier caso, es en nombre de la cientificidad del proyecto antropológico que se opera la disyunción. Etnografía, comparatismo, ambición de realizar una ciencia del espíritu humano: el programa era suficientemente estimulante para justificar que se aceptara una buena dosis de positivismo y que se sacrificasen las viejas lunas filosóficas.

Por su parte, Foucault expresó en Las palabras y las cosas el interés que le producía la antropología. Nótese primero que propone, al final de ese trabajo de "arqueología de las ciencias humanas", en el último capítulo de la obra, una definición de estas últimas que pone el acento sobre su relación con el inconsciente: "Se dirá, pues, que hay "ciencia humana" no por todas aquellas partes en que se trata del hombre, sino siempre que se analiza, en la dimensión propia de lo inconsciente, las normas, las reglas, los conjuntos significativos que develan a la conciencia las condiciones de sus formas y de sus contenidos" (1968: 354). En este sentido, psicoanálisis y etnología encarnan la quintaesencia de esas ciencias, pues tienen en común ser "ambas ciencias del inconsciente" (ibíd.: 367). La etnología hace visible detrás de las representaciones de los hombres "las normas a partir de las cuales los hombres realizan las funciones de la vida, pero rechazando su presión inmediata, las reglas a través de las cuales experimentan y mantienen sus necesidades, los sistemas sobre el fondo de los cuales les es dada cualquier significación" (id.). La fuerza propia de la etnología, como la del psicoanálisis, es "lo que Lévi-Strauss dijo de la etnología: que disuelven al hombre" (ibíd.: 368). La pertinencia de la etnología no viene de que trate sociedades sin historia, sino de que se interese ante todo por los "procesos inconscientes que caracterizan el sistema de una cultura dada" (ibíd.: 368).
Lo que fascina a Foucault es la posibilidad de acceder así al "sistema de los inconscientes culturales [es decir] el conjunto de estructuras formales que harían significativos los discursos míticos, darían su coherencia y su necesidad a las reglas que rigen las necesidades" (id.), y el hecho de que la lingüística pueda proporcionar un modelo formal a la etnología y al psicoanálisis. El entusiasmo del filósofo por las ciencias humanas encuentra claramente su fuente en la lectura de Lévi-Strauss y de Freud revisitado por Lacan. Foucault, como Louis Althusser en la misma época, ve en el desarrollo de la etnología y del psicoanálisis preciosos aliados en su estrategia por establecer un verdadero "antihumanismo teórico" y promover una epistemología crítica que tematizará algunos años más tarde La arqueología del saber. No es pues sin razón que a estos autores se les atribuyera la etiqueta de "estrucuturalistas".

$Y$, sin embargo, un poco como la etiqueta "nouveau roman" había permitido promover autores de los que se puede rápidamente medir sus diferencias, la etiqueta "estructuralismo" también estará sujeta a malentendidos. A pesar de Las palabras y las cosas, ni Lévi-Strauss ni Foucault han reivindicado jamás ninguna proximidad. En los años 1970, no encontramos en este último sino muy pocas referencias a Lévi-Strauss y más generalmente a la antropología. ¿Es asunto de desconocimiento o simple falta de interés? ¿Hay que ver ahí una reticencia más profunda? Según mi conocimiento, sólo un texto evoca temáticamente esta disciplina. Se trata de una conferencia pronunciada en la Facultad de Filosofía de la Universidad de Bahía en 1976, llamada "Las mallas del poder". Ese texto es realmente notable, pues nos permite comprender la distancia que separaba a Foucault de la antropología y, particularmente, de la obra de Lévi-Strauss. La conferencia de Bahía se inicia con una crítica global del psicoanálisis y de las ciencias sociales enfocando la noción de ley. Los psicoanalistas "continúan considerando todavía en sus trabajos que el significado del poder, el núcleo central, aquello en que consiste el poder, sigue siendo la prohibición, la ley, el hecho de decir no, y una vez más la fórmula "no debes" (Foucault, 1999: 236). Comparten con los psicólogos y los sociólogos una concepción del poder esencialmente negativa y ante todo jurídica. El poder se identifica con el espacio de la regla y de la prohibición: "Me parece que esta concepción fue formulada incisivamente y desarrollada ampliamente por la etnología, a finales del siglo XIX" (ibíd.: 236). Según Foucault, esta disciplina no ha en efecto dejado de identificar sistema de poder y sistema de reglas. Emblemáticos de este enfoque, los trabajos de Lévi-Strauss y su interés por el problema del incesto colocan en el corazón de la investigación la cuestión de la prohibición. Es interesante notar que, para Foucault, la antropología de Lévi-Strauss, que inscribe en la línea durkheimiana, es de lado a lado política en tanto transmite una teoría del poder. Ciertamente su objeto explícito es el parentesco y la alianza, pero el filósofo no busca evaluar la pertinencia de ello en este dominio y deja de lado la reelaboración levistraussiana de los conceptos de intercambio y reciprocidad. La crítica de Foucault se 
refiere a la imposición de la regla y su envés, lo prohibido. Se tiene allí una concepción extremadamente restrictiva del poder que no hace sino prolongar el discurso jurídico que ha dominado las teorías del Estado en Occidente desde la Edad Media. El poder está esencialmente representado en el derecho, y así se elaboró la noción de soberanía que hoy en día sigue siendo un concepto mayor de la teoría política. A esta juridización (juridicisation) ${ }^{2}$ de lo político, Foucault opone un análisis de los mecanismos positivos del poder. En contraste con las concepciones dominantes centradas sobre el binomio regla/prohibición, nota la aparición en "años más recientes" de "nuevos puntos de vista" (ibíd.: 237): un punto de vista "estrictamente marxista", un punto de vista "más alejado del marxismo clásico". Y cita a Pierre Clastres que desarrolla, según él, "una nueva concepción del poder como tecnología" (id.). Foucault no especifica esta referencia, e indica más adelante en su conferencia que él mismo se inspiró en Marx para elaborar su propia teoría de los mecanismos de poder, de las tecnologías disciplinarias y de la perspectiva biopolítica. Volveré sobre estos diferentes puntos, pero señalo aquí que encontramos en ese texto una lectura de Marx que pone el acento sobre la importancia del tomo II del Capital en el que Foucault encuentra varias ideas clave: en primer lugar, no hay uno sino varios poderes; luego, estos poderes no derivan de un poder central; además, estos poderes no tienen por función principal impedir, ellos son "productores de una eficacia, de una aptitud, productores de un producto" (ibíd.: 240). La disciplina del taller, la disciplina en la armada, ilustran esta positividad del poder. Para pensar el poder, los teóricos políticos privilegiaron un objeto, el Estado y sus aparatos. Para salir del impase de la juridicidad (juridisme), es pues a la vez a una lectura del Marx analista del universo industrial, y a un rebasamiento de la antropología estructural hacia otras perspectivas, que Foucault apela. Se puede conjeturar que lo que le interesaría en la obra de Clastres sería la idea de que puedan existir sociedades diferentes de las nuestras, que no se acomodan al poder en su dimensión coercitiva. Aunque Foucault ve en la obra de Clastres emerger una noción de poder como tecnología, podemos, sin embargo, preguntarnos si este antropólogo que subraya sin cesar la negatividad de toda fuerza coercitiva, considera como el filósofo que el poder tiene una positividad. La conferencia de Bahía no fue publicada en Francia en vida de su autor y ningún debate comprometió verdaderamente a Foucault y los antropólogos alrededor de las cuestiones que él había planteado. ¿Lo deseaba él mismo? En ausencia de evidencia a este respecto, no me arriesgaré a responder a la pregunta.

Durante el mismo período, la antropología norteamericana mucho tiempo dominada por los partidarios del culturalismo entraba en crisis. Crisis intelectual y política. Al debilitamiento de los conceptos que habían asegurado el éxito de la disciplina, empezando por la noción misma de cultura que venían a sacudir las críticas provenientes

\footnotetext{
${ }^{2} \mathrm{~N}$. del T. Introducimos este neologismo para indicar la reducción de la noción de poder a un concepto jurídico. Mantenemos la palabra en francés para que el lector pueda acompañar sus resonancias.
}

de la antropología estructural y de la teoría marxista, se añadía una reflexión cada vez más crítica sobre la práctica misma de la antropología. Esta tenía por punto partida el contexto de la guerra de Vietnam, y el hecho de que la etnografía hubiera podido ser utilizada para fines militares y que ciertos antropólogos hubieran sido instrumentalizados por el poder establecido. Esta situación suscitó un cuestionamiento profundo, primero sobre la noción de objetividad y los criterios hasta entonces admitidos en materia de cientificidad en la disciplina; luego, a propósito de las condiciones mismas de la práctica etnográfica. Si se refiere a los debates de la época, se disciernen dos momentos: por un lado la discusión que se desarrolla sobre el tema "antropología e imperialismo" en el seno de la Asociación Antropológica Americana (American Anthropological Association), que da lugar a textos en los que el eco internacional fue considerable ${ }^{3}$; por otro lado, un conjunto de reflexiones epistemológicas sobre la descripción etnográfica (Geertz, 1973) y la posición del antropólogo como observador y como autor (Clifford \& Marcus, 1986; Geertz, 1988).

Es, entonces, a finales de los años 1970 que se produce en la antropología norteamericana un movimiento profundo que se caracteriza al mismo tiempo por una impugnación de los presupuestos sobre los que la disciplina había funcionado hasta entonces y por la búsqueda de perspectivas teóricas y terrenos nuevos. Ahora bien, en la misma época Foucault hace su entrada en la escena académica norteamericana: conferencias, traducciones, publicaciones de fragmentos escogidos le aseguran rápidamente un prestigio considerable. Es interesante observar que uno de los introductores de Foucault en los Estados Unidos no es otro que Paul Rabinow, él mismo antropólogo y antiguo alumno de Clifford Geertz. Él es el editor de Foucault Reader (1984), uno de los textos más difundidos en las universidades, y de una obra sobre Foucault escrita en colaboración con Hubert Dreyfus (1984), que presenta también las respuestas del filósofo a las preguntas de los dos autores. De regreso de su primer trabajo de campo en Marruecos, Rabinow (1977) había elaborado un conjunto de reflexiones a propósito de la práctica de la etnografía. Sus críticas se referían específicamente a la concepción tradicional del trabajo de campo, como aislado, desconectado de un contexto fuertemente marcado por el colonialismo, y a las relaciones ambiguas que se instauran entre el etnógrafo y sus fuentes.

Los trabajos de Rabinow se inscriben en una corriente más vasta que va a contribuir a la renovación de la antropología en los Estados Unidos a partir de los años 1980. Esta corriente procede a un cuestionamiento radical de aquello que Geertz (1986) ha denunciado como la "autoridad del etnógrafo". Esta procura estar ante todo fundada sobre la experiencia ("yo estaba allí"), y el texto etnográfico se dirige a lograr que el lector se identifique con el punto de vista del observador participante. Hay pues una toma de poder inaugural que prohíbe toda forma de reflexión en

\footnotetext{
${ }^{3}$ Fueron editados en francés por Jean Copans (1975).
} 
lo concerniente a las condiciones de la experiencia a la que apela el teórico-investigador. La mayoría de las veces, este último se hace el modesto, pretendiendo borrarse completamente detrás de los "datos" recogidos sobre el terreno. De ahí un cierto tipo de escritura de la etnografía que procede entre un vaivén de generalidades y casos concretos. En ningún momento se encuentran interrogadas las condiciones etnográficas de la experiencia. El seminario de Santa Fe, que reúne en 1984 a James Clifford, George Marcus, Paul Rabinow y otros jóvenes antropólogos, tomó precisamente como tema la etnografía como género literario.

Negándose a dar por sentadas las pretensiones de la antropología de ser una ciencia, los trabajos salidos del seminario (Clifford \& Marcus, 1986) desembocaban en un cuestionamiento de la autoridad del etnógrafo-autor. Como lo ha escrito más tarde Rabinow: "Writing Cultures no presentó una visión del mundo. Hizo algo diferente articuló una problematización emergente. El concepto de problematización proviene de Michel Foucault, quien lo definió así: 'El desarrollo de lo dado en una cuestión, esta transformación de un grupo de obstáculos y dificultades en problemas a los cuales diversas soluciones tratan de ofrecer una respuesta'" 1999: 307). No es casual que se refiera aquí a Foucault. Desde los años 1980, el filósofo francés interesaba a la nueva generación de antropólogos norteamericanos, y esto por dos razones. Por un lado, él había mostrado el carácter histórico y relativo de la división operada entre ciencia y literatura. Por otro, Foucault no había dejado de plantear la cuestión de la verdad y de su relación con el poder, como continuación del pensamiento nietzscheano. Writing Cultures se inscribe en la perspectiva corrosiva propia del proyecto genealógico propuesto por Nietzsche y actualizado por Foucault.

Es esta puesta en evidencia de las estrategias discursivas en su relación con el poder la que ha atraído particularmente a los antropólogos norteamericanos cuando descubrieron a Foucault. En la época, podían leer principalmente una antología de textos y entrevistas de Foucault sobre las relaciones entre poder y saber. Es, pues, el encuentro entre la reflexión del Foucault "arqueólogo" y las preocupaciones de los antropólogos cada vez más dubitativos respecto del positivismo que había prevalecido en su universo de conocimiento, el que va a caracterizar este período. Al otro lado del Atlántico, lo que preocupa a los partidarios de la nueva antropología es reencontrar una base epistemológica modificando de algún modo el enfoque de la etnografía, a riesgo de hacer vacilar la figura del sabio que se había impuesto en esta disciplina. De la misma manera, el descubrimiento de Derrida va a jugar un rol muy importante en esta iniciativa de desestabilización de la ortodoxia etnográfica. Todo esto no se da sin contradicción, ya que por ejemplo Geertz, que está en el origen de la corriente crítica en antropología, se refiere ante todo a la tradición hermenéutica, particularmente al filósofo francés Paul Ricoeur. La consigna geertziana "tratar la cultura como un texto" implica como correlato la idea de que el texto es ante todo materia y cuestión de interpretación. Ahora bien, Derrida, por su parte, destruye la noción de interpretación que se inscribe, según él, en la tradición más pura de la metafísica occidental. Si la referencia a Derrida y a Foucault va ha hacerse cada vez más frecuente, los antropólogos no buscan por ello definir la relación que mantienen con esos textos filosóficos.

Se puede decir que en los años 1980 se constituyó un cierto estilo de relación entre los antropólogos norteamericanos y la filosofía "continental": una relación referencial (y un poco reverencial). Son pocos los textos que no hacen referencia en algún momento de una página (generalmente en la introducción) a Foucault. Esto no significa que se interrogue verdaderamente sobre el propósito de este último. Esto vale también para Derrida, la tendencia era aquí asimilar la deconstrucción con un método de lectura, a riesgo de acompañar con ello los considerandos del positivismo borrando el carácter subversivo de la obra del filósofo. Sin demorarnos sobre los malentendidos que ha podido suscitar la recepción de Foucault o Derrida, mantenemos por el momento un punto positivo: la apertura de la antropología a la reflexividad. Deleuze escribía: "El principio general de Foucault es el siguiente: toda forma es un compuesto de relaciones de fuerza" (1987: 159). Todo pasa como si fuera ante todo esta dialéctica de la fuerza y de la forma que había retenido los antropólogos preocupados de pasar por el tamiz de la autocrítica las producciones discursivas procedentes de su disciplina.

Una obra publicada en la misma época por George Marcus y Michel Fischer (1986), dos de los pioneros de Santa Fe, refleja bien esta voluntad de dotar a la antropología de un alcance crítico, mostrando cómo el concepto de cultura y las etnografías que se lo reclaman han devenido instrumentos al servicio del imperialismo. Desde las primeras páginas, estos autores coinciden con la crítica del orientalismo elaborada por Edward Said y, a lo largo del texto, muestran la necesidad de restaurar la función crítica de la antropología. Esto implica el desarrollo de una etnografía reflexiva teniendo en cuenta en su propio discurso las condiciones de construcción de su objeto. Esto necesita también la aplicación de una crítica de nuestra propia cultura, a través de las estrategias de "desfamiliarización". De ahí que ponga fin a la gran división entre los Otros (los exóticos, objetos de la etnografía) y Nosotros (los Occidentales, los científicos). Lo que está en juego es claro: terminar con una concepción de la ciencia de las culturas juzgada perimida y, sobre todo, redefinir el campo de investigación de una antropología cuya intención crítica es en adelante explícita. En ese contexto, el recurso a autores como Jean Baudrillard, Walter Benjamin, Pierre Bourdieu, Jacques Derrida, Michel Foucault, Jean-François Lyotard, el interés por los trabajos procedentes de los estudios poscoloniales, tales como los de Homi Bahbha, Partha Chaterjee, Edward Said, Gayatri Spivak, van a contribuir a reconfigurar el terreno de una antropología que no duda en estar del lado de la 
perspectiva posmodernista refiriéndose a David Harvey (1990) y a Fredric Jameson (1984).

Esta apertura de la antropología a una problematización epistemológica y filosófica apenas tuvo eco al otro lado del Atlántico. En la época, sólo algunos textos de Clifford Geertz son traducidos al francés; la reflexión crítica de James Clifford sobre la escritura de la etnografía encuentra cierto eco (Études rurales, 1985), pero, por lo demás, se tiende sobre todo a burlar la deriva de la antropología norteamericana en la cual se une el epíteto fácilmente peyorativo de posmoderno. Curiosamente, en el momento en que los trabajos de Foucault y de los pensadores franceses posestructuralistas comienzan a interesar en sumo grado a la antropología norteamericana, no encuentran casi eco en esta disciplina en Francia. ¿Es decir que la antropología no habría conocido una evolución considerable en este último país? ¿O que habría quedado impávida, insensible a cualquier interrogación filosófica?

De hecho, estamos aquí en plena paradoja: en el momento mismo en que aparecían los grandes textos de Foucault, los antropólogos franceses (Georges Balandier, Michel Izard, Pierre Clastres, Marc Augé, Emmanuel Terray, Maurice Godelier, etc.) se apasionaban por el problema del poder. Pero no se encuentra en la época ningún esbozo de diálogo, incluso de controversia, con el autor de La voluntad de saber. La antropología prefiere nutrirse de sus propios terrenos y recurrir a los conceptos que ella se ha forjado. En cambio, los filósofos, en este caso Gilles Deleuze y Félix Guattari, pero también Claude Lefort, Marcel Gauchet, apelan a los trabajos de los antropólogos en su reflexión sobre la política. Antes incluso que la antropología norteamericana haya tomado el giro que sabemos, es en Francia que se encuentran las denuncias más argumentadas de compromisos de la etnología con el colonialismo y de la esencialización de lo "primitivo" y de lo "arcaico", que ha contribuido a perder de vista la realidad histórica y política en la que opera el campo etnográfico. Sin embargo, aun allí no se encuentra ninguna alusión a las investigaciones foucaultianas sobre los "regímenes de verdad", la producción de saberes y los modos de discursividad. Se funda en un principio simple: es posible para el antropólogo hacer emerger al final de sus investigaciones una verdad que escapa a los actores sociales, enredados en representaciones necesariamente parciales de la realidad. Que se lean las reflexiones de Lévi-Strauss sobre los conceptos de modelo y estructura, la lectura propuesta por Maurice Godelier de los análisis marxistas del fetichismo, o Bosquejo de una teoría de la práctica de Pierre Bourdieu. Nada pone en duda el télos de una ciencia del hombre orientada por el ideal de la verdad.

Si al inicio de los años 1980 los antropólogos franceses y norteamericanos convergen en la crítica al imperialismo y sus efectos, y acuerdan sobre la necesidad de interrogarse respecto de la posición del observador, se produce una desconexión que se debe en gran parte al impacto al otro lado del Atlántico de la reflexión foucaultiana sobre el orden del discurso y las complejidades del poder y del saber. Posteriormente la distancia crecerá, al punto que la referencia a Lévi-Strauss termina por ser eclipsada en los Estados Unidos en provecho de la invocación de los posestructuralistas, mientras que del lado francés se observa un endurecimiento de una antropología que parece comprometida a defender sus territorios y sus temas tradicionales (parentesco, simbolismo, ritual, etc.).

La cuestión del poder en los términos en que la planteaba el autor de Vigilar y Castigar no podía por tanto dejar indiferente a la antropología de lo político. Y eso por dos razones. En primer lugar, Foucault planteaba claramente que "el análisis en términos de poder no debe postular, como datos iniciales, la soberanía del Estado, la forma de la ley o la unidad global de una dominación; éstas son más bien formas terminales" (1998: 112). Antes de él, la antropología política había contribuido a des-reificar la política, mostrando hasta qué punto la polarización sobre la forma estatal había terminado por ocultar la diversidad real de las figuras del poder. En Anthropologie de l'État (Abélès, 2005), he señalado esta convergencia, que parece sin embargo no haber tocado en la época a otros antropólogos ${ }^{6}$.

Además, Foucault insistía sobre la necesidad de problematizar el "cómo" del ejercicio del poder. Pensar el poder en acto, como "un modo de acción sobre acciones" (Foucault, 1991: 84), era también rechazar los instrumentos tradicionales de teorías políticas que habían "recurrido a maneras de pensar el poder basadas en modelos legales, es decir: ¿qué legitima el poder?, o basadas en modelos institucionales, es decir: ¿qué es el Estado?" (Foucault, 1991: 52). Esto significaba desjuridizar (déjuridiciser) y desinstitucionalizar (désinstitutionnaliser) nuestra aproximación a lo político. Ahora bien, este camino se me ha presentado muy sugestivo en el momento en el que intentaba explorar en Europa un universo que parecía $a$ priori reacio a un modo de análisis constituido por pensar lo político en las sociedades llamadas "sin Estado". Los trabajos que hemos realizado sobre las prácticas políticas, en contextos tan diferentes como el entorno aldeano o la Unión Europea, tienen en común esta voluntad de identificar el "cómo" del poder, sus ramificaciones, sus redes. La necesidad de una aproximación deliberadamente no institucionalista de las instituciones se ha impuesto.

Una pregunta focal en esta perspectiva es la del gobierno de los hombres: llegamos aquí a un tema caro a Foucault, el del arte de gobernar, es decir de las prácticas por las cuales se llega a "estructurar el campo de acción posible de los otros" (Foucault, 1991: 86). Pues una de las dificultades que encuentra toda antropología del poder mantiene el aspecto permanente que se opera entre el "arte de gobernar" -conjunto de procedimientos concretos que diseñan el campo de relaciones de fuerzas-y un discurso de la soberanía que pretende a la vez fundar la legitimidad de estos procedimientos y desplegar la significación, a riesgo de postular un horizonte de trascendencia, un más allá del 
poder. El fenómeno de la realeza divina en las sociedades africanas ofrece un buen ejemplo del ajuste del poder en una metafísica de la soberanía.

En lo que concierne a la política en las sociedades "cercanas", la articulación entre poder y discurso de la soberanía es tan fuerte que sólo un trabajo de "desfamiliarización" puede permitir poner en evidencia la diferencia, como Foucault ha intentado hacerlo, haciendo jugar a la historia el rol de un operador que permite a la vez proporcionar una mirada alejada sobre el poder contemporáneo y poner al día los datos constitutivos. En los siglos XVII y XVIII se inventa, según él, una nueva mecánica de poder que se ejerce sobre los cuerpos y no más sobre la tierra. Ahora bien, la teoría clásica de la soberanía está ligada a un poder que se ejerce ante todo sobre la tierra y sus productos: "es una teoría que permite fundar el poder en torno y a partir de la existencia física del soberano y no de los sistemas continuos y permanentes de vigilancia" (Foucault, 2008: 43-44). Paradójicamente, el principio de soberanía va a perdurar incluso cuando se desarrolla lo que Foucault llama "una mecánica polimorfa de la disciplina (ibíd.: 45). Se tendrá entonces una organización jurídica articulada alrededor del principio de soberanía que coexistirá con una mecánica disciplinaria.

Foucault no aceptó nunca pensar esta cuadrícula disciplinaria en términos de represión o prohibición. Criticando a Wilhelm Reich y Pierre Legendre ("el amor del censor"), indica claramente que hay todavía allí una reducción de los procedimientos de poder a una problemática jurídica anclada en la soberanía, lo que induce, entre otras cosas, una concepción del poder como negatividad, como productor de prohibición ("el poder, es lo que dice no" [ibíd.: 423]). El desplazamiento que opera Foucault lo lleva a desplegar en su plasticidad las relaciones de poder, mostrar hasta qué punto están ellas mismas imbricadas en otro tipo de relaciones, que ellas son multiformes y locales, de ahí la noción de micropoderes. Desde el punto de vista de la etnografía que se interesa en situaciones localizadas, que se sumerge en lo cotidiano, esta noción, lejos de resultar anodina, ha inspirado los trabajos de Michel de Certeau y su equipo. De Certeau no duda en designar las actividades cotidianas como tácticas. Tácticas, pues no localizables en términos de aparato de campo social, sin proyecto global. La realidad observada por el etnógrafo se asemeja a un hormigueo de relaciones.

En su investigación sobre las tecnologías de poder, Foucault no se contenta con poner al día la aparición de técnicas disciplinarias centradas sobre el individuo y su cuerpo; muestra también cómo se puso en práctica a fines del siglo XVIII una nueva tecnología que trata a la multiplicidad de hombres, a la población. Es lo que nombra la biopolítica, que trata de la población como problema a la vez científico y político. El interés dado a la demografía, el desarrollo de la higiene pública, las instituciones de asistencia y seguridad, la consideración de las relaciones entre el hombre y el medio ambiente, diseñan una configuración nueva en la que la dimensión disciplinaria se difumina para dar lugar a un proyecto apuntando a prolongar la vida, regularizar los mecanismos biológicos. Con el ascenso del capitalismo, estas tecnologías disciplinarias del trabajo van a ver aumentar los procedimientos menos directos permitiendo una "toma de poder" sobre los hombres como grupo de seres vivos. El hombre no es más sólo sujetado en su singularidad de individuo, es igualmente controlado como espécimen de una población de seres vivos: la población como entidad indivisa de vivos es el nuevo sujeto de la soberanía biopolítica.

Mientras que las técnicas disciplinarias se aplicaban especialmente al hombre concebido en su individualidad corporal, el hombre-cuerpo, las técnicas biopolíticas incorporan la multiplicidad de los hombres como masa global focalizándose sobre el hombre-especie. Al contrario de la disciplina que se mantenía en una "anatomopolítica", la biopolítica designa la toma de control por el poder de los procesos que afectando la vida, desde el nacimiento hasta la muerte (enfermedad, vejez, discapacidad, efectos del medio, etc.), y que, para ser absolutamente aleatorios a escala individual, tienen, como fenómeno colectivo, efectos económicos y políticos determinantes. El nacimiento de la ciencia policial y las premisas de las "políticas" de salud pública han situado progresivamente la vida biológica o natural entre las preocupaciones técnicas de gestión, los cálculos y las previsiones del Estado. Es mucho menos la conformidad del estilo de vida y las costumbres de los sujetos políticos lo que preocupa al Estado que su nacimiento, la inscripción en los registros políticos de la nacionalidad y de la demografía de su "vida biológica".

En la escala biopolítica el individuo no es más tenido como objetivo, es considerado por la norma biopolítica como espécimen de una población de la que hay que regular los movimientos, tanto internos como externos: disminución, crecimiento, desplazamiento. Al contrario de la soberanía tradicional, caracterizada por el poder de hacer morir y dejar vivir, el poder se define en adelante por su capacidad de hacer vivir y dejar morir. Lejos de estar en contradicción con esta redefinición del poder orientado hacia la vida, el racismo de Estado, y los millones de muertos que ha producido, se afirma él mismo como parte interesada de esta problemática de "fortalecimiento biológico" (Foucault, 2008: 233) de una población.

Es interesante observar que la problemática del biopoder ha inspirado directamente investigaciones recientes en antropología. A propósito de la mundialización, ha dado mucho que hablar la imbricación cada vez más estrecha de lo local y lo global. Más importantes me parecen las interrogaciones que han tratado el paradigma del Estado-nación que dominó el siglo XX, estremecido por la emergencia de nuevos modos de gubernamentalidad infra o supra-nacional. Lo que está en juego aquí es el modelo de la soberanía territorial, y coincide con los análisis de Foucault cuando subraya que, en adelante, el poder ya no tiene una afinidad particular con el territorio sino que se 
ejerce principalmente sobre las multiplicidades. Pues bien, en el espacio transnacional diseñado por la globalización, la movilidad, los desplazamientos, lo que Arjun Appadurai (1996) designa como flujos, juegan un rol esencial. Es lo que explica la focalización de lo político sobre la cuestión de la inmigración, las catástrofes humanitarias y el problema de los refugiados.

Para pensar el mundo desterritorializado, no es sorprendente que los antropólogos recurran a los conceptos foucaultianos, en la medida en que ofrecen una luz nueva sobre situaciones complejas. Los trabajos sobre las diásporas lo testimonian. Un ejemplo: el caso de los chinos de Hong Kong, tal como lo analiza Aihwa Ong (1999). En tiempos de la dominación británica, eran considerados ciudadanos de territorios británicos dependientes (British Dependant Territory Citizens, $B D T C)$; tenían derecho de viajar, pero no de residir en Gran Bretaña. Desde la transferencia de Hong Kong a China son ciudadanos británicos de ultramar (British National Overseas) y se benefician de lo que Ong llama una ciudadanía parcial (partial citizenship). Siempre han sido distinguidos como gente de color (colored people), con el mismo título que los afro-caribeños, pero gozan de un tratamiento particular en razón de su dinamismo económico. En 1990, el gobierno británico cambia su política de inmigración a fin de conceder la ciudadanía a ciertos residentes chinos de Hong Kong. Se trataba ante todo de evitar un movimiento de pánico ligado a la transferencia a China, lo que habría podido desestabilizar por largo tiempo el mercado. Es así que 50.000 residentes chinos de Hong Kong, miembros de la élite, y sus familias obtuvieron la nacionalidad británica. Se había seleccionado gente aún joven, de 30 a 40 años, con un alto nivel de educación y que por sus actividades y sus redes participaran en actividades del capital transnacional. Para Ong, los cambios sucesivos de las autoridades de Londres respecto de la ciudadanía reflejan la aplicación de una biopolítica que trata de manera diferenciada a sectores de población según su ubicación en el dispositivo económico.

Otra línea de investigación abierta por la reflexión sobre la biopolítica concierne a la condición de las poblaciones despojadas de toda inscripción territorial e institucional, los que son reducidos al estado de "nuda vida" (nuta vita), según la fórmula de Giorgio Agamben (1997) que pretende extender la reflexión de Foucault. Es esta vida desnuda desconectada del contexto cívico que hace la "materia" de las masas de refugiados, residentes nociudadanos instalados de manera permanente o provisoria sobre territorios de estados industrializados, en los guetos de las ciudades o en los campos de refugiados. He ahí lo que prefigura, según Agamben, una forma de comunidad sin territorio y sin frontera cuyo estatuto de refugiado actual anuncia la constitución venidera. Objeto de una inseguridad jurídica en cuanto a su identidad o su ciudadanía, el estatus de refugiado, que conocen masas crecientes de población, disloca el tríptico Estado-naciónterritorio heredado de la época clásica y pone en juego otra definición de la relación sujeto/ soberanía: "el espacio de la vida desnuda, situado en el origen al margen del ordenamiento, viene progresivamente a coincidir con el espacio político" (Agamben, 1995: 12).
La temática de la vida desnuda ha conocido estos últimos años un cierto eco en antropología. Esto se debe al hecho de que, de Kosovo a Ruanda, los investigadores ponen en práctica una etnografía de los conflictos, de la violencia y sus efectos. La experiencia del sufrimiento, la miseria, la negación de humanidad en esas situaciones extremas (Malkki, 1995; Kleinman, Das \& Lock, 1997; Agier, 2002), pero también en el contexto de la marginalización del que son víctimas los que son abandonados en los márgenes de la ciudad (sin papeles, excluidos, etc.), ha dado lugar a reflexiones que se sostienen sobre el discurso y la práctica por los que la gubernamentalidad contemporánea trata la vida (Fassin \& Memmi, 2004). En una perspectiva idéntica, los trabajos consagrados a los márgenes del Estado (Das \& Poole, 2004), a esos espacios políticos donde su control se encuentra permanentemente cuestionado y donde las fronteras entre la ley y la fuerza devienen borrosas. Se mide aquí la influencia de una lectura de Foucault que vuelve a centrar la cuestión de la gubernamentalidad sobre la biopolítica, la gestión de las poblaciones, las tecnologías centradas sobre lo vivo y la reconfigura a través de una reflexión sobre la soberanía y el estado de excepción. En este contexto, las periferias, las minorías aparecen como el objeto por excelencia de una antropología que quiere asumir plenamente un rol crítico respecto de las formas poscoloniales de la dominación. Al mismo tiempo, si se observa más de cerca, se trata sin embargo de una interpretación basada sobre ciertos textos de Foucault (particularmente la última lección del curso de 1976) que pone el acento en la articulación entre tanatopolítica y biopolítica y que sitúa en su centro el concepto de soberanía. Ella encierra lo político en las redes de lo jurídico y, apoyándose en los análisis de Carl Schmitt, desemboca en una disimetría fundamental entre un dominio de normatividad donde prima la represión y un espacio de indeterminación donde sólo les queda a los humanos su vida desnuda: el espacio de la ciudad adosado al soberano que deviene la fuente de todo poder, para empezar por el de decretar la excepción, es por excelencia un espacio de exclusión. Llevado al extremo, este esquema desemboca en la asimilación de la gubernamentalidad a una mecánica totalitaria frente a la cual los sujetos son reducidos al estado de cuerpos sufrientes. En cierta medida, la antropología no hace sino reencontrar el espacio tradicional que le había sido asignado en el campo de las ciencias sociales: focaliza sus investigaciones sobre situaciones de alteridad y exclusión. Si puede vanagloriarse de estar del lado de los dominados, no acepta menos implícitamente el juridismo que Foucault denunciaba rechazando el concepto de soberanía y la reducción del poder a la pura negatividad de lo que reprime y de lo que prohíbe, "un poder esencialmente negativo que supone por una parte un soberano cuyo papel es el de prohibir y por otra un sujeto que debe de algún modo decir sí a esta prohibición" (Foucault, 1979: 169).

La antropología pierde de vista entonces lo que sin duda hay de más corrosivo en el aporte teórico y político del filósofo, la idea de sostener el análisis sobre "el poder en sus mecanismos positivos" (Foucault, 1999: 239), lo que implica que se restituya la complejidad propia de la gubernamentalidad, que se tomen en cuenta las transformaciones que la afectan. Nunca se insistirá lo suficiente sobre la necesidad para la antropología 
política, especialmente en el contexto de la globalización (Abélès, 2008), de intensificar sus investigaciones sobre dispositivos de gobierno que rediseñan progresivamente la configuración de las relaciones entre lo vivo y lo civil. Cierto reduccionismo haciendo hincapié en la referencia a la biopolítica tiene como consecuencia por un lado subestimar la dimensión propiamente conceptual y filosófica de la obra de Foucault apropiándose de un aspecto de la elaboración teórica y de la problematización del concepto de gubernamentalidad que concierne a la gestión de las poblaciones, mientras que él despliega toda una reflexión sobre lo económico y más particularmente sobre la génesis del liberalismo. Por otro lado, no se puede subestimar el hecho de que el horizonte de Foucault ha sido el del Estado-providencia y la crisis que lo afectaba. Ahora bien, el momento del Estado-providencia ha resultado de la adecuación entre dos tipos de poder, el poder pastoral y el poder político que se ejercen, uno sobre los "individuos vivos" y el otro sobre los "sujetos civiles". $Y$ hoy no es casualidad si dos dominios se impusieron en el corazón del dispositivo de poder transnacional, este de los intercambios económicos y ese de lo humanitario. Importa pensar esta nueva situación y la emergencia de la "política global" en que la autonomización relativa suscita tensiones nuevas, sus iniciativas tienden permanentemente a desestabilizar el gobierno estatal existente. Entre los temas de investigación que se esbozan está el relativo a la economía de subsistencia y a la manera en que paralelamente a su inversión del campo humanitario, al margen de los conflictos, los órganos trasnacionales y las ONG que reclaman su anclaje en la "sociedad civil" vienen a su modo a complejizar y perturbar el juego reconectando los dominios que los gobiernos habían cuidadosamente dividido distinguiendo la acción económica y las iniciativas relacionadas con la urgencia humanitaria (Abélès, 2006). No es necesario ser un experto para percibir hasta qué punto la oposición entre política y sociedad civil es poco operativa, pues las ONG que se encuentran ellas mismas en una relación de representación respecto de sus mandatarios son partes interesadas de una relación de fuerza y buscan ejercer un poder, un "modo de acción sobre acciones", para retomar la fórmula de Foucault (1991: 84). Lo que nos interesa es la manera en que la impugnación y las diversas formas de resistencia encuentran una expresión en este tejido difuso y ramificado de organizaciones.

Los trabajos realizados por los antropólogos sobre los conflictos armados y la intervención humanitaria permiten penetrar mejor esta dimensión de la política. Los trabajos recientes (Duffield, 2001; Pandolfi, 2002) muestran bien cómo la injerencia humanitaria suscita una verdadera inversión de parte de órganos transnacionales que desarrollan estrategias específicas e intentan extender su control sobre las instituciones locales. En principio, son considerados como apolíticos, pero su acción se inscribe en una relación de fuerzas que ellos contribuyen a modelar, con la participación más o menos interesada de los grupos que localmente se instituyen como sus interlocutores legítimos. Se desarrolla una configuración de poderes cada vez más desterritorializados donde los aparatos tradicionales del Estado-nación ya no tienen un rol directo sobre un real profundamente modificado por el conflicto.

Desde un punto de vista más global, lo que está en juego hoy es un conjunto de procesos que nos obligan a reestructurar profundamente una conceptualización de lo político anclada, articulada alrededor del concepto de soberanía. Proponiendo un enfoque que se podría designar como una pragmática de la gubernamentalidad, esta última entendida como "conjunto de acciones sobre acciones posibles", Foucault abría una pista sin ninguna duda fecunda. Los que intentan llevar la antropología política al terreno de las formas emergentes, como yo he sido llevado a hacerlo a propósito de la construcción europea, se encuentran en efecto ante el siguiente dilema: o se unen al institucionalismo dominante y se resignan a ignorar los problemas planteados por la construcción teórica de su objeto, aplastándolo bajo conceptos que terminan por desnaturalizarlo; o aceptan tener que vérselas con "objetos políticos no identificados" según la expresión de Jacques Delors. Esta postura que me parece, a la larga, la única sostenible es la, preconizada por Foucault, del "empirista ciego", el investigador que tantea y que ensaya fabricar instrumentos sin demasiada consideración por los conformismos disciplinarios.

\section{*}

Si no hay que despachar la tarea demasiado rápido, como se ha tenido a veces tendencia a hacerlo, transponiendo sin precaución en todos los dominios sus análisis de lo biopolítico, retengamos el legado más precioso del filósofo, cuando nos incita a volver interminablemente sobre la cuestión del poder, a perseguir allí los más pequeños arcanos. Más allá de los debates sobre la adecuación de la categoría de biopoder para dar cuenta de nuestra contemporaneidad, debate lejos de estar hoy cerrado, hay que subrayar la perspectiva epistemológica significativa que se abre en la estela de Foucault, de pensar la antropología política como una pragmática de la gubernamentalidad. Asumir un camino tal quiere decir continuar forjando instrumentos para aprehender mejor los desplazamientos que afectan lo político y que demuestra la reconfiguración de las relaciones de poder. Pero, ¿no es indispensable, frente a la complejidad de los procesos políticos, mostrarse audaces e imaginativos en nuestros tanteos?

\section{Bibliografía}

Abélès, M. (2005, 1990). Anthropologie de l'État. Paris, Payot. [2e éd.].

Abélès, M. (2006). Politique de la survie. Paris, Flammarion.

Abélès, M. (2008). Anthropologie et globalisation. Paris, Payot.

Abélès, M. \& M. Cuillerai (2002). “Mondalisation: du géoculturel au biopolitique", Anthropologie et Sociétés, 26 (1): 11-28.

Agamben, G. (1995). Moyens sans fins. Notes sur la politique. Paris, Rivages.

Agamben, G. (1997). Homo Sacer, 1. Le pouvoir souverain et la vie nue. Paris, Le Seuil.

Agamben, G. (1999). Ce qui reste d'Auchwitz. Paris, Rivages.

Agier, M. (2002). Aux bords du monde, les réfugiés. Paris, Flammarion. 
Appadurai, A. (1996). Modernity at Large. Minneapolis, University of Minnesota Press.

Clifford, J. \& G. Marcus (eds.) (1986). Writing Cultures. Berkeley, University of California Press.

Copans, J. (ed.) (1975). Anthropologie et impérialisme. Paris, Maspéro.

Das, V. \& D. Poole (eds.) (2004). State and its Margins: Comparative Ethnographies. Santa Fe, School of American Research Press / Oxford, James Currey.

Deleuze, G. (1987). Michel Foucault. José Vásquez Pérez (trad.). Barcelona, Paidós.

Derrida, J. (1986 [1967]). De la gramatología. Oscar del Barco y Conrado Ceretti (trads.). México, Siglo XXI.

Dreyfus, H. \& P. Rabinow (1984). Michel Foucault. Un parcours philosophique. Paris, Gallimard ("Folio Essais").

Dreyfus, H. \& P. Rabinow (2001). Michel Foucault. Más allá del estructuralismo y la hermenéutica. Rogelio C. Paredes (trad.). Buenos Aires, Nueva Visión.

Duffield, M. (2001). Global Governance and the New Wars: The Merging of Development and Security. London, Zed Books.

Études Rurales (1985). Étude Rurales 97-98: Le texte ethnographique. Éd. par Françoise Zonabend et Jean Jamin. Paris, Éd. de l'EHESS.

Evans-Pritchard, E. E. \& M. Fortes (eds.) (1940). African Political Systems. Oxford, Oxford University Press.

Evans-Pritchard, E. E. y Meyer Fortes (eds.) (2010). Sistemas políticos africanos. México, CIESAS, Universidad Autónoma Metropolitana, Universidad Iberoamericana.

Fassin, D. \& D. Memmi (eds.) (2004). Le Gouvernement des corps. Paris, Éd. de l'EHESS.

Foucault, M. (1968). Las palabras y las cosas. Una arqueología de las ciencias humanas. Elsa Cecilia Frost (trad.). México, Siglo $\mathrm{XXI}$.

Foucault, M. (1969). L'Archéologie du savoir. Paris, Gallimard.

Foucault, M. (1979). Microfísica del poder. Julia Varela y Fernando Álvarez Uría (trads). Madrid: Ediciones la Piqueta.

Foucault, M. (1980). Power/Knowledge. Selected Interviews and Writings, 1972-1977. New York, Pantheon Books.

Foucault, M. (1984). "Questions et réponses", in Hubert Dreyfus \& Paul Rabinow, Michel Foucault. Un parcours philosophique. Paris, Gallimard ("Folio Essais").

Foucault, M. (1991). El sujeto y el poder. Edgar Garavito (trad.). Bogotá, Carpe Diem.
Foucault, M. (2008). Defender la sociedad. Horacio Pons (trad.). Buenos Aires, Fondo de Cultura Económica.

Foucault, M. (2001, 1994). Dits et Écrits, II: 1976-1988. Paris, Gallimard.

Foucault, M. (1998). Historia de la sexualidad I. La voluntad de saber. Ulises Guiñazú (trad.). México, Siglo XXI.

Foucault, M. (1999). Obras esenciales, III: Estética, ética y hermenéutica. Ángel Gabilondo (trad.). Barcelona, Paidós.

Foucault, M. (2012). El poder, una bestia magnífica. Sobre el poder, la prisión y la vida. Horacio Pons (trad.). Buenos Aires, Siglo XXI.

Geertz, C. (1973). The Interpretation of Cultures. New York, Basic Book. [Trad. franç. partielle: Bali. Interprétation d'une culture, Paris, Gallimard, 1984.]

Geertz, C. (1988). Works and Lives: The Anthropologist as Author. Stanford, Stanford University Press. [Trad. franç.: Ici et là-bas. L'anthropologue comme auteur, Paris, Métailié, 1992.]

Harvey, D. (1990). The Condition of Postmodernity. Oxford, Blackwell.

Jameson, F. (1984). "Postmodernism or the Cultural Logic of Capitalism", New Left Review 146: 53-92.

Kleinman, A. V. Das \& M. Lock (eds.) (1997). Social Suffering. Berkeley, University of California Press.

Malkki, L. (1995). Purity and Exile. Chicago, University of Chicago Press.

Marcus, George (ed.) (1999). Critical Anthropology Now. Santa $\mathrm{Fe}$, School of American Research Press.

Marcus, G. \& M. Fischer (eds.) (1986). Anthropology as Cultural Critique. Chicago, University of Chicago Press.

Ong, A. (1999). Flexible Citizenship. The Cultural Logics of Transnationality. Duke, Duke University Press.

Pandolfi, M. (2002). "'Moral entrepreneurs', souverainetés mouvantes et barbelés. Le biopolitique dans les Balkans postcommunistes", Anthropologie et Sociétés 26 (1): 29-51.

Rabinow, P. (1977). Reflections on Fieldwork in Morocco. Berkeley, University of California Press. [Trad. franç.: Un ethnologue au Maroc, Paris, Hachette, 1988.]

Rabinow, P. (1999). "American Moderns", in George Marcus, ed., Critical Anthropology Now. Santa Fe, School of American Research Press: 305-333.

Said, E. (1979). Orientalism. New York, Random House. [Trad. franç.: L'Orientalisme, Paris, Le Seuil, 1980.] 